\title{
Study and Comparison of Constellation diagrams of 16-QAM in different fading channels
}

\author{
Abhishek Goyal, Dr. Anubhuti Khare \\ Dept. of Electronics \& Communication EngineeringUniversity Institute of Technology RGPV \\ Bhopal, India 462033 \\ Dept. of Electronics \& Communication EngineeringUniversity Institute of Technology RGPV \\ Bhopal, India 462033
}

\begin{abstract}
In this paper constellations for 16-quadrature amplitude modulation (16-QAM) in Rayleigh and Nakagami-m fading channels is studied. These constellation diagrams are useful for visualizing the effect of fading to the transmitted signal symbols. We simulated 16-QAM technique at different SNR and draw the constellation diagrams for Rayleigh and Nakagami-m fading channels for different values of $m$. At lower SNR the constellations are more dispersed. It shows that the effect of fading is more dominated at lower SNR. When the SNR is increased the effect of fading is reduced and it can be seen as constellations are less dispersed. We compare the BER versus SNR plot for AWGN and Rayleigh channel and also for AWGN and Nakagami-m fading channels for different values of $m$. It shows that the when we increase the value of $m$ (nakagami factor) then the effect of fading increases and we need more SNR for reducing this effect of fading.
\end{abstract}

Index Terms: 16-QAM, additive white Gaussian noise (AWGN), fading channels, constellation, BER, SNR

\section{Introduction}

When we deal with wireless communication, then the environment, surroundings and terrain profiles between transmitter and receiver are the important factors that play an important role in wireless system design. Signal propagates in multiple paths and due to this multipath propagation receiver do not receive the exact signal which was transmitted and strength of the signal is weakened down and also some noise is added. Because each signal goes through multiple path and each path add some amplitude and phase attenuation due to terrain profile, so the received signal is the superposition of the signal through all multiple path. This results either constructive or destructive interference of the signal. This is known as fading. There is need to estimate the effect of these factors on a wireless system and then develop a mathematical model. [1]

In $\mathrm{M}-\mathrm{QAM}$ modulation technique, the constellation size $\mathrm{M}=2^{\mathrm{b}}$, where $\mathrm{b}$ is the number of bits transmitted. It means that in M-QAM techniques M symbols transmitted and each symbol transmits $b=\log 2(\mathrm{M})$ bits. We use 16-QAM modulation technique, it means that the constellation size $\mathrm{M}=16$ and no of bits transmitted by each 16 symbols is $b=\log 2(16)$ i.e. $b=4$ bits.

In 16-QAM there are 16 symbols and each symbol transmit 4 bits ranging from binary 0000 to binary 1111.It means we transmitted 16 symbols consisting 4 binary bits of different amplitude and phase difference. A symbol is comprised of in-phase and quadrature-phase component. So we draw the constellation diagram of the transmitted symbols between the in-phase and quadrature-phase component to study the behavior of fading in the case of Rayleigh and nakagami-m fading channels with different values of nakagami factor (m).[2]

In this paper the constellations for 16-QAM technique is investigated in the case of Rayleigh and Nakagami-m fading channels using a combination of theory and simulation. For 16-QAM simulation 1000000 bits are transmitted and then calculate the bit error rate in Rayleigh and Nakagami-m fading channels with different values of $\mathrm{m}$. Graphs are drawn between the BER and the SNR required to transmit the signal. For constellation diagrams 100 errors are calculated and then draw constellation diagrams between in-phase and quadrature-phase component. It is then shown that more disperse constellation diagram serves more fading at lower SNR. When we increase the value of SNR, constellations are less dispersed and BER reduced.

\section{System model}

We consider 16-QAM technique in Rayleigh and nakagami-m fading channel with different values of nakagami factor $(\mathrm{m})$. In our system model we assume that there is no direct line-of-sight (LOS) path between transmitter and receiver.

We consider 16-QAM technique. The sampled baseband signal $r(t)$ is given by [3]

$$
r(t)=a e^{j \beta} s(t)+n(t)
$$

Where $\mathrm{a}=$ fading amplitude

$\beta=$ phase of signal uniformly distributed between 0 to $2 \pi$

$\mathrm{n}(\mathrm{t})=$ zero mean circularly symmetric complex Gaussian noise i.e. $\mathrm{E}\left[\mathrm{nn}^{*}\right]=0$. 
$s(t)=$ transmitted symbol chosen from $\left\{\sqrt{E_{s}} e^{j 2 \pi x}\right\}_{x=0}^{M-1}$ with symbol energy $E_{s}$.

We use 16-QAM modulation technique it means that constellation size $M=16$, so the transmitted symbol chosen from $\left\{\sqrt{E_{\mathrm{s}}} \mathrm{e}^{\mathrm{j} 2 \pi \mathrm{x}}\right\}_{\mathrm{x}=0}^{15}$.Transmitted signal goes through various multiple paths. Due to this multipath propagation there is fluctuation and attenuation in amplitude of signal. Because the channel fading amplitude varying randomly due to the configuration of fading channels, so it is a random variable and it requires some distribution for channel modeling. In received signal, the channel fading amplitude may change with some distributions like as Rayleigh, Nakagami-m.

When the communication environment is so dense or obstructed then there is no direct line-of-sight (LOS) path. In this case when no direct LOS path is present, then the Rayleigh distribution can be used. According to Simon and Alouini [4], in Rayleigh fading distribution the channel fading amplitude a is distributed as

Where $\mathrm{a}=$ fading amplitude

$$
P_{a}(a)=\frac{2 a}{\omega} \exp \left(-\frac{a^{2}}{\omega}\right), a \geq 0
$$

$\omega=\overline{a^{2}}$ (mean square value of fading amplitude) environment.

$\mathrm{P}_{\mathrm{a}}(\mathrm{a})=$ Probability density function which is dependent on the nature of the radio propagation For this Rayleigh fading distribution instantaneous signal-to-noise power ratio $(\sigma)$ per symbol is calculated as

Where $\mathrm{E}_{\mathrm{s}}=$ energy per symbol

$$
\sigma=\frac{\mathrm{a}^{2} \cdot \mathrm{E}_{\mathrm{s}}}{\mathrm{N}_{0}}
$$

$\mathrm{N}_{0}=$ power spectral density $(\mathrm{W} / \mathrm{Hz})$

Thus the average SNR $(\bar{\sigma})$ per symbol is given as

$$
\bar{\sigma}=\frac{\omega E_{s}}{N_{0}}
$$

The instantaneous SNR $(\sigma)$ per symbol of the Rayleigh fading channel is distributed according to an exponential distribution given by

$$
\mathrm{P}_{\sigma}(\sigma)=\frac{1}{\bar{\sigma}} \exp \left(-\frac{\sigma}{\bar{\sigma}}\right), \quad \sigma \geq 0
$$

The nakagami-m probability distribution is given by

Where $\mathrm{a}=$ fading amplitude,

$$
\mathrm{P}_{\mathrm{a}}(\mathrm{a})=\frac{2 \mathrm{~m}^{\mathrm{m}} \mathrm{a}^{2 \mathrm{~m}-1}}{\Gamma(\mathrm{m}) \omega^{\mathrm{m}}} \exp \left(-\frac{\mathrm{ma}^{2}}{\omega}\right), \mathrm{a} \geq 0
$$

$\mathrm{m}=$ nakagami parameter also called shape parameter on which the shape of nakagami- $\mathrm{m}$ distribution depends and it ranges $\frac{1}{2}$ to $\infty$.

$\omega=$ A parameter that controlling spread

The mathematical formulas of parameter $\mathrm{m}$ and $\omega$ given by

$$
\begin{gathered}
\mathrm{m}=\frac{\mathrm{E}^{2}\left[\mathrm{a}^{2}\right]}{\operatorname{Var}\left[\mathrm{a}^{2}\right]} \\
\omega=\mathrm{E}\left[\mathrm{a}^{2}\right]
\end{gathered}
$$

Where $\mathrm{E}\left[\mathrm{a}^{2}\right]$ expectation of fading amplitude and $\operatorname{Var}\left[\mathrm{a}^{2}\right]$ is the variance of fading amplitude.

\section{Simulation Results And Discussion}

In this section we present simulation results for the 16-QAM in the case of Rayleigh and nakagami-m fading channels with different values of $\mathrm{m}$. In simulation, we have modeled the received signals by generating Rayleigh and nakagami variables for different values of $m$ and then compare both with AWGN and calculate BER.

In Fig.1 constellation of transmitted symbols in case of the Rayleigh fading channel is shown at SNR 6 DB. Fig. 1 shows that constellations are overlapping each other and more dispersed. It means that the effect of fading is more dominated at 6 DB SNR and BER is order of $10^{-1}$.

Description of Fig.2 is same as the Fig. 1 except this is for 10 DB SNR. In Fig.3 and Fig.4 the constellations are less dispersed in comparison of previous figures and obtained BER in order of $10^{-2}$. Fig.5 and 
Fig. 6 obtained BER in order of $10^{-3}$.Fig. 7 and Fig.9 obtained BER in order of $10^{-4}$ and $10^{-5}$ respectively. In Fig. 11 and Fig.12 there is very less dispersion, it means that fading is very less and we able to obtain the BER of order $10^{-6}$. It is clear from the above figures when the SNR is increased the constellations are less dispersed and it means that the effect of fading is reduced and thus result lower BER.

Fig. 12 shows a comparison of BERs of 16-QAM between both AWGN and Rayleigh fading channel. It is clearly seen in Fig. 12 that AWGN channel requires less SNR around 11 DB to achieve the BER of order $10^{-6}$ as compare to Rayleigh fading channel which requires more SNR around 54 DB to achieve same BER.

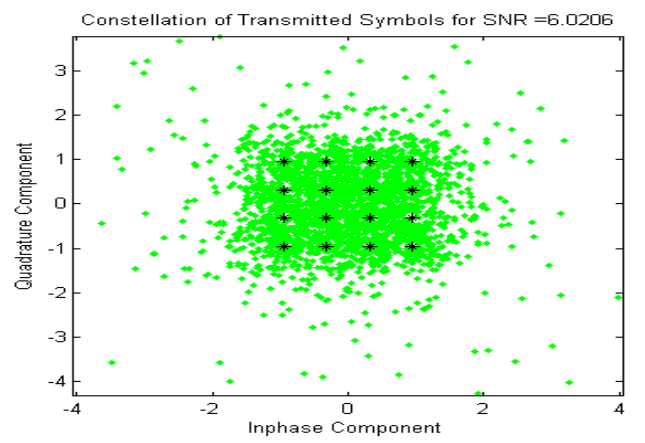

Fig.1 (Constellation of transmitted symbols in case of Rayleigh channel for SNR 6 DB for 16-QAM)

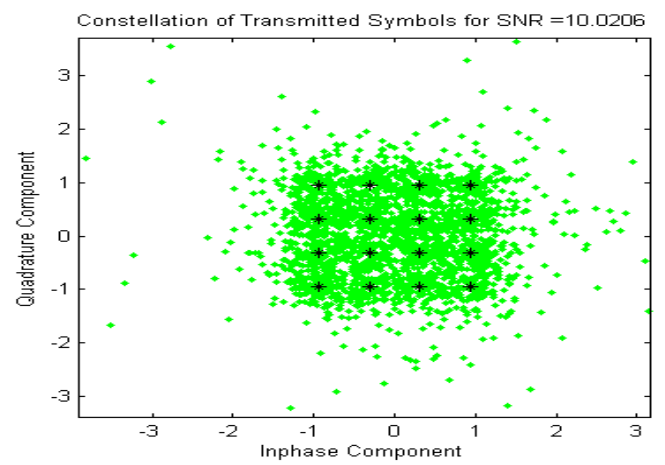

Fig.2(Constellation of transmitted symbols in case of Rayleigh channel for SNR 10 DB for 16-QAM )

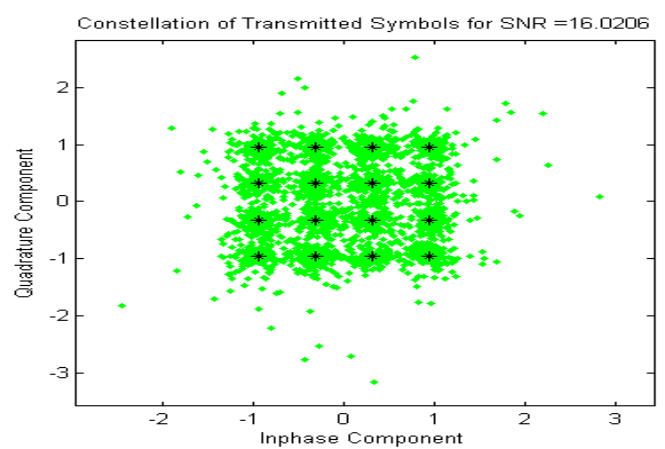

Fig.3(Constellation of transmitted symbols in case of Rayleigh channel for SNR 16 DB for 16-QAM) 


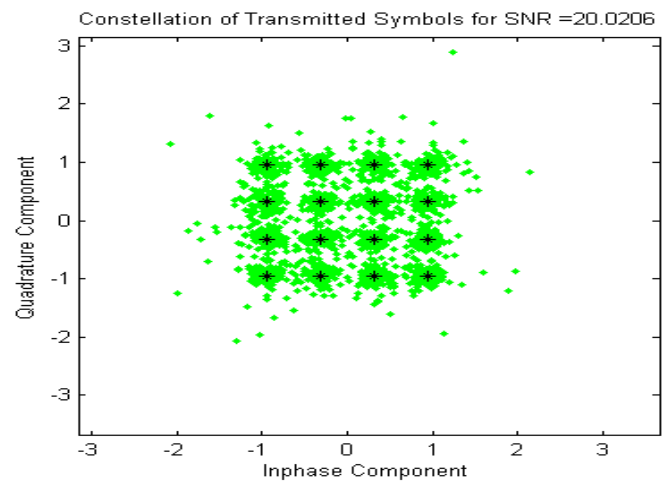

Fig.4(Constellation of transmitted symbols in case of Rayleigh channel for SNR 20 DB for 16-QAM )

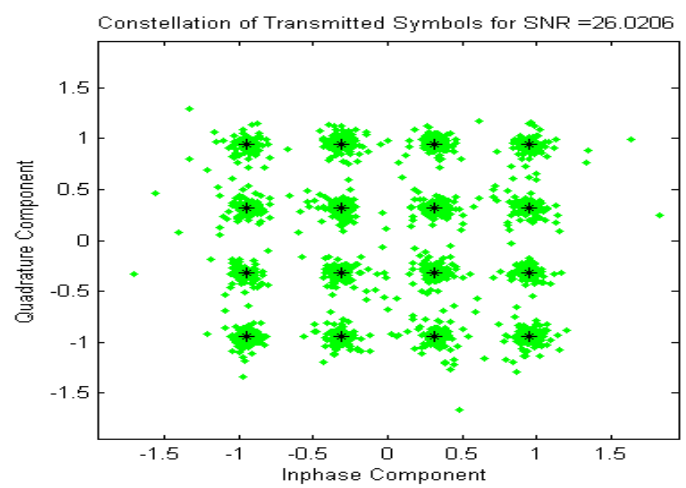

Fig.5(Constellation of transmitted symbols in case of Rayleigh channel for SNR 26 DB for 16-QAM)

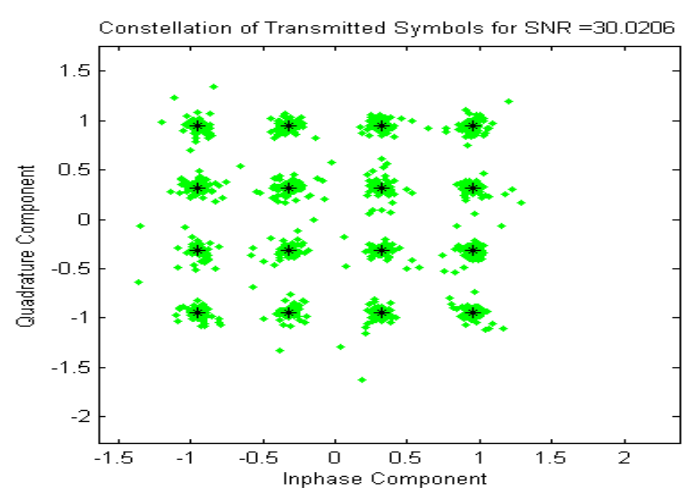

Fig.6(Constellation of transmitted symbols in case of Rayleigh channel for SNR 30 DB for 16-QAM )

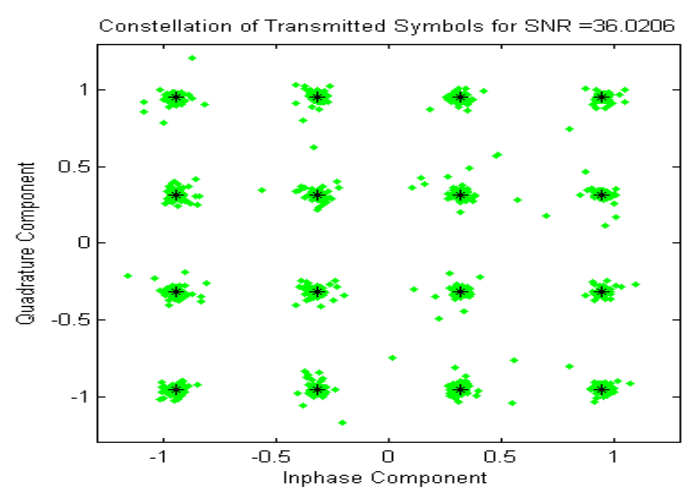

Fig.7(Constellation of transmitted symbols in case of Rayleigh channel for SNR 36 DB for 16-QAM ) 


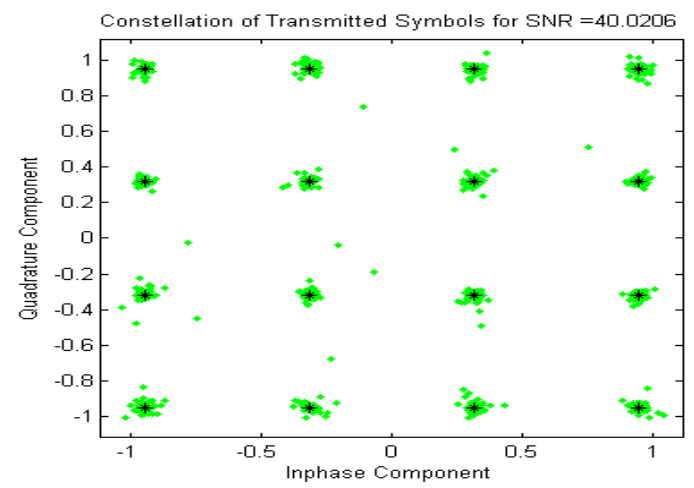

Fig.8(Constellation of transmitted symbols in case of Rayleigh channel for SNR 40 DB for 16-QAM)

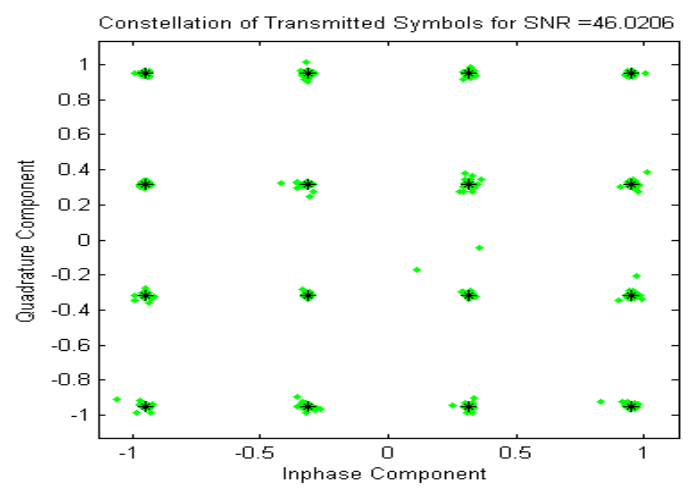

Fig.9(Constellation of transmitted symbols in case of Rayleigh channel for SNR 46 DB for 16-QAM)

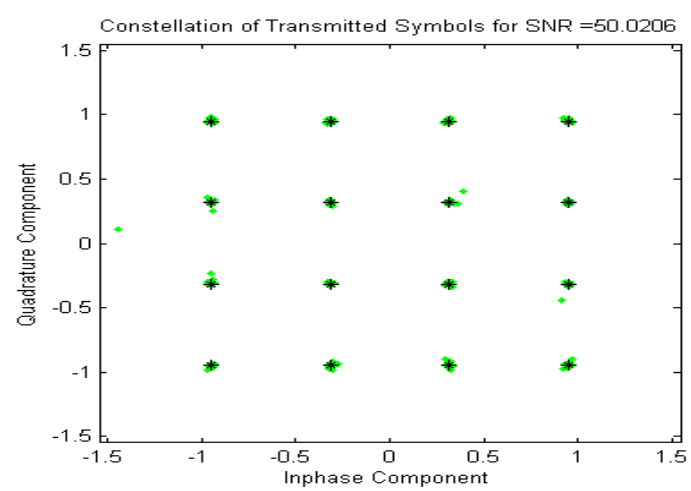

Fig.10(Constellation of transmitted symbols in case of Rayleigh channel for SNR 50 DB for 16-QAM)

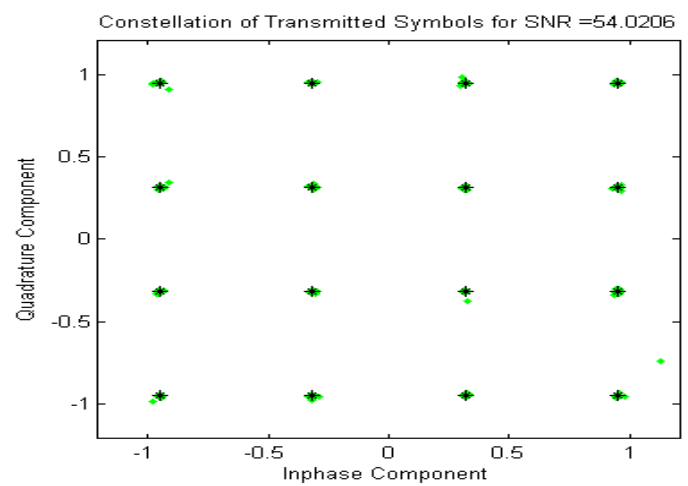

Fig.11(Constellation of transmitted symbols in case of Rayleigh channel for SNR 56 DB for 16-QAM) 


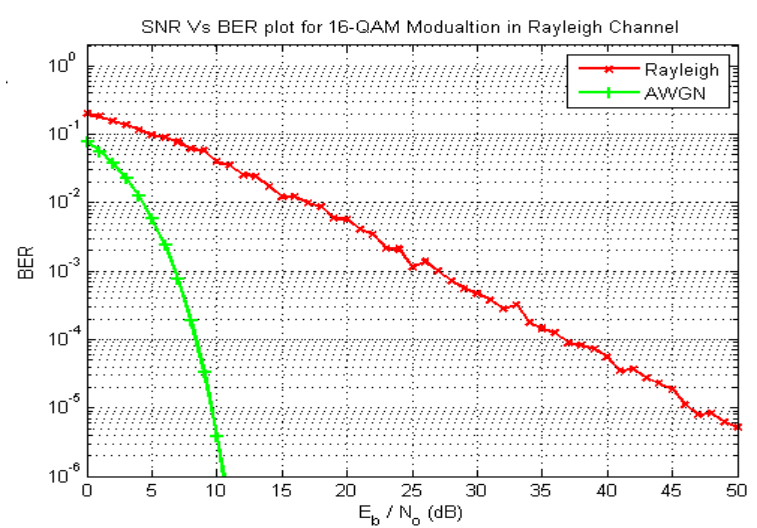

Fig.12(Comparison of BER between AWGN and Rayleigh channel for 16-QAM)

Fig. 13 shows the constellation of transmitted symbols for nakagami-m fading channel with value of nakagami factor $m=1$ at SNR 6 DB and achieves BER of order $10^{-2}$.

Fig. 14 shows the constellation at SNR $10 \mathrm{DB}$ and achieves BER of order $10^{-3}$. Fig. 15 shows the constellation at SNR 16 DB and achieves BER of order $10^{-6}$. However, at SNR 16 DB BER is of order $10^{-6}$ but transmitted signal is still faded so we need more SNR to improve the effect of fading. At 22 DB SNR the effect of fading less with compare to 16 DB SNR and the constellation are less spread, it can be seen in Fig.16.

Fig. 17 shows Comparison of BER between AWGN and Nakagami-m channel with $m=1$ for 16-QAM .It shows that we need 5 DB more SNR in case of nakagami-m fading channel with $m=1$ to achieve the same BER of AWGN.

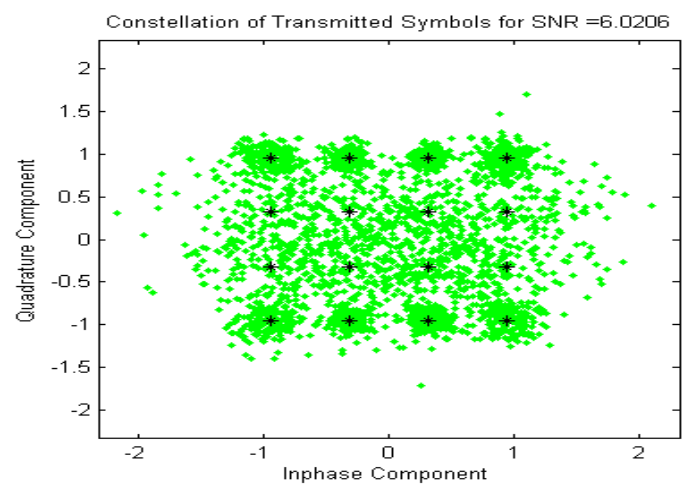

Fig.13(Constellation of transmitted symbols in case of Nakagami-m channel with $m=1$ for SNR 6 DB for 16-

QAM)

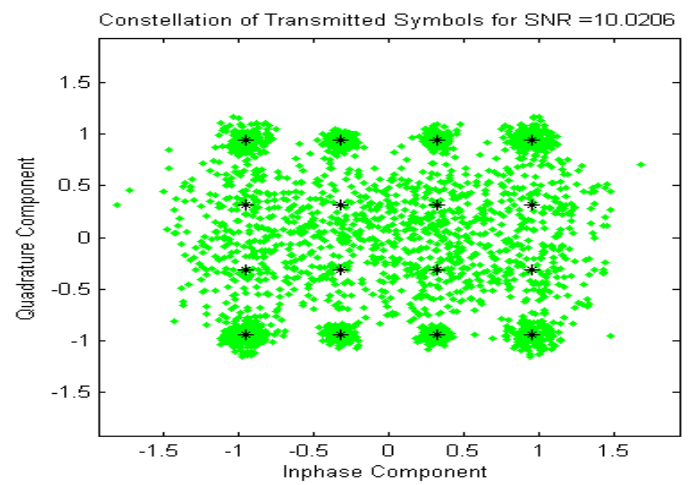

Fig.14(Constellation of transmitted symbols in case of Nakagami-m channel with $m=1$ for SNR 10 DB for 16-

QAM ) 


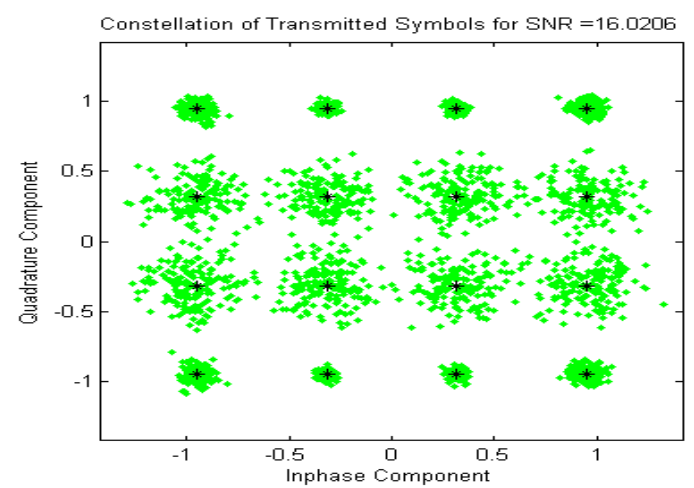

Fig.15(Constellation of transmitted symbols in case of Nakagami-m channel with $m=1$ for SNR 16 DB for 16QAM )

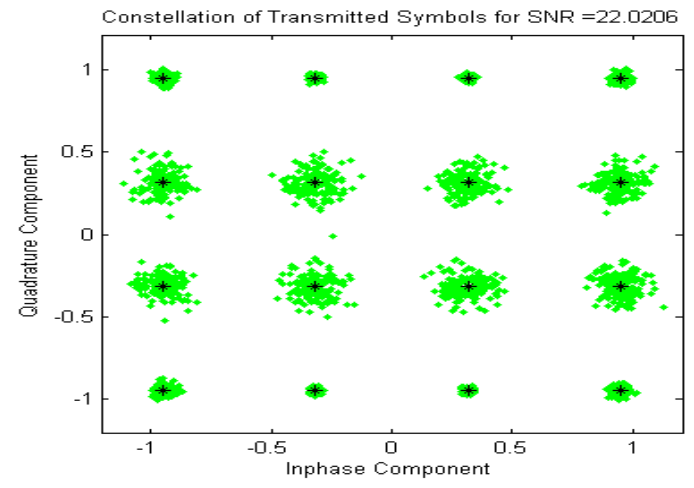

Fig.16(Constellation of transmitted symbols in case of Nakagami-m channel with $m=1$ for SNR 22 DB for 16QAM )

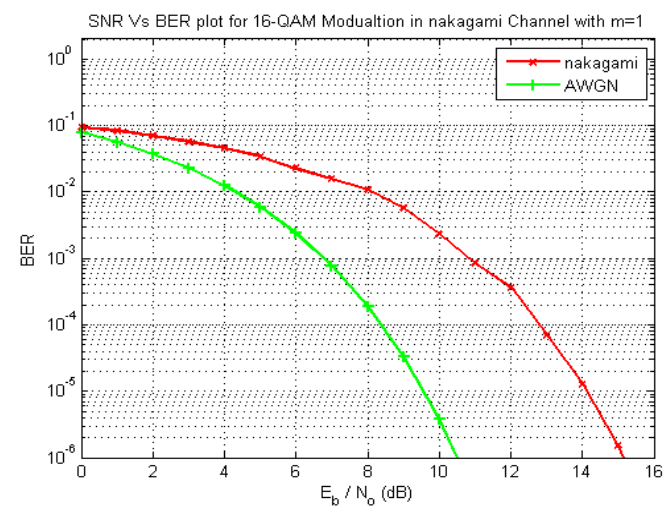

Fig.17(Comparison of BER between AWGN and Nakagami-m channel with $\mathrm{m}=1$ for 16-QAM)

Fig. 18 shows the constellation of transmitted symbols for nakagami-m fading channel with value of nakagami factor $\mathrm{m}=2$ at SNR $6 \mathrm{DB}$ and achieves BER of order $10^{-2}$.

Fig. 19 shows the constellation at SNR 10 DB and achieves BER of order $10^{-2}$.Fig.20 shows the constellation at SNR 16 DB and achieves BER of order $10^{-5}$. However at SNR 16 DB BER is of order $10^{-5}$ but transmitted signal is still faded so we need more SNR to improve the effect of fading. In Fig.21 the constellations at SNR 22 DB are shown, it can be seen that at 22 DB SNR the effect of fading less with compare to 16 DB SNR and the constellations are less spread.

Fig.22 shows Comparison of BER between AWGN and Nakagami-m channel with $\mathrm{m}=2$ for 16-QAM .It shows that we need 7 DB more SNR in case of nakagami-m fading channel with $m=2$ to achieve the same BER of AWGN. 


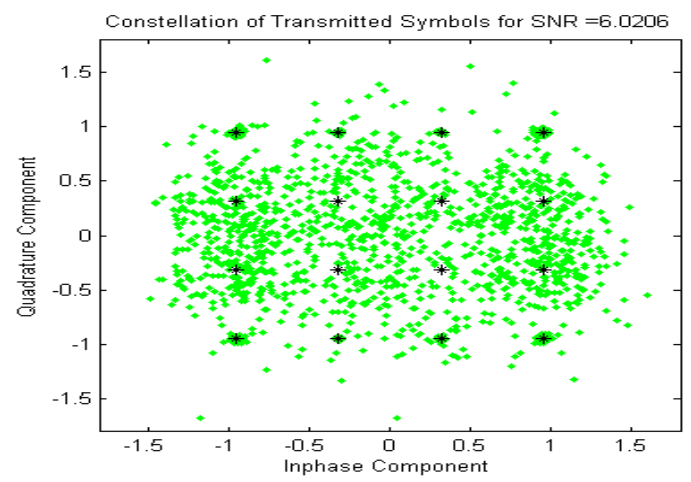

Fig.18(Constellation of transmitted symbols in case of Nakagami-m channel with $\mathrm{m}=2$ for SNR 6 DB for 16QAM )

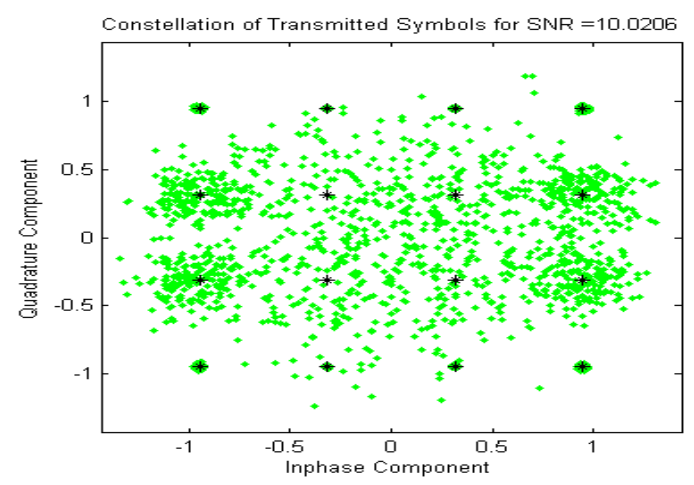

Fig.19(Constellation of transmitted symbols in case of Nakagami-m channel with $m=2$ for SNR10 DB for 16QAM)

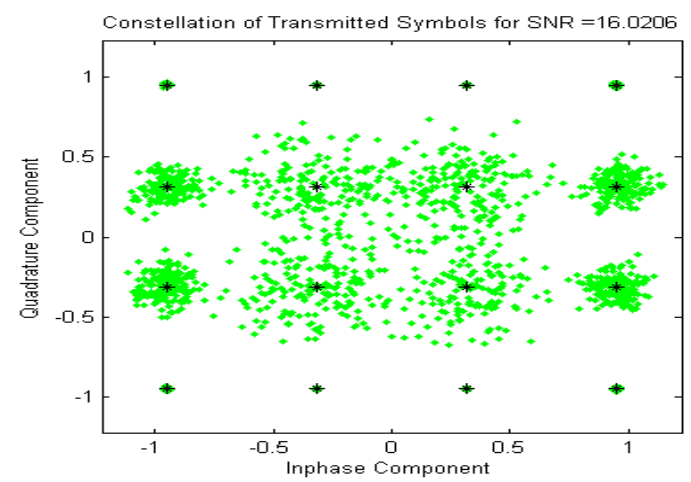

Fig.20(Constellation of transmitted symbols in case of Nakagami-m channel with $\mathrm{m}=2$ for SNR16 DB for 16QAM)

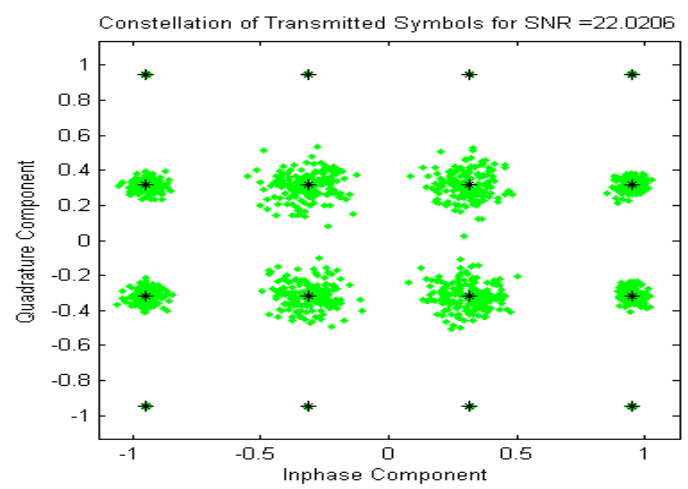

Fig.21(Constellation of transmitted symbols in case of Nakagami-m channel with $m=2$ for SNR 22 DB for 16QAM) 


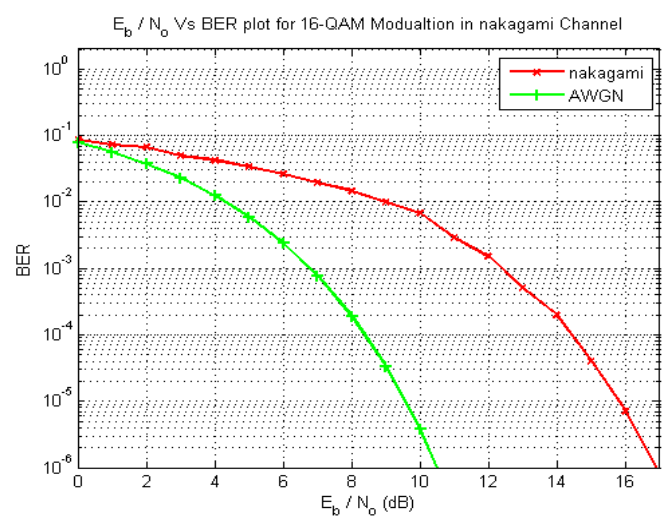

Fig.22(Comparison of BER between AWGN and Nakagami-m channel with $\mathrm{m}=2$ for 16-QAM)

Fig. 23 shows the constellation of transmitted symbols for nakagami-m fading channel with value of nakagami factor $\mathrm{m}=3$ at SNR $6 \mathrm{DB}$ and achieves BER of order $10^{-1}$.

Fig.24 shows the constellation at SNR 10 DB and achieves BER of order $10^{-2}$.Fig. 25 shows the constellation at SNR $16 \mathrm{DB}$ and achieves BER of order $10^{-3}$.Fig.26 shows the constellation at 24 DB SNR. However at SNR 24 DB BER is of order $10^{-6}$ but transmitted signal is still faded.

Fig.27 shows Comparison of BER between AWGN and Nakagami- $m$ channel with $m=3$ for 16-QAM .It shows that we need $10 \mathrm{DB}$ more SNR in case of nakagami-m fading channel with $\mathrm{m}=2$ to achieve the same BER of AWGN.

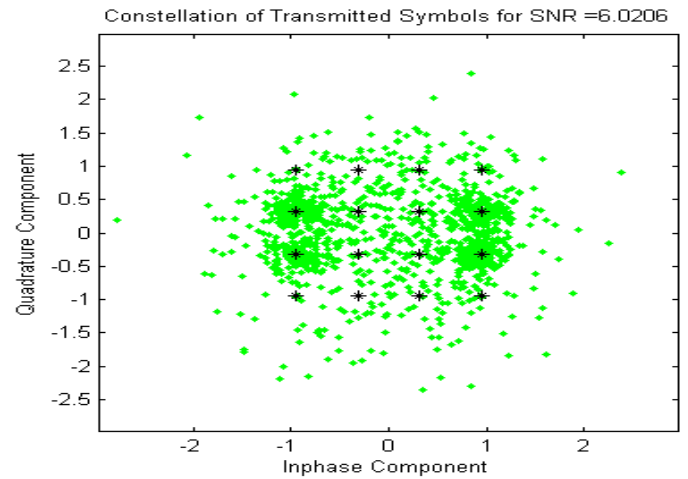

Fig.23(Constellation of transmitted symbols in case of Nakagami-m channel with $m=3$ for SNR 6 DB for 16QAM)

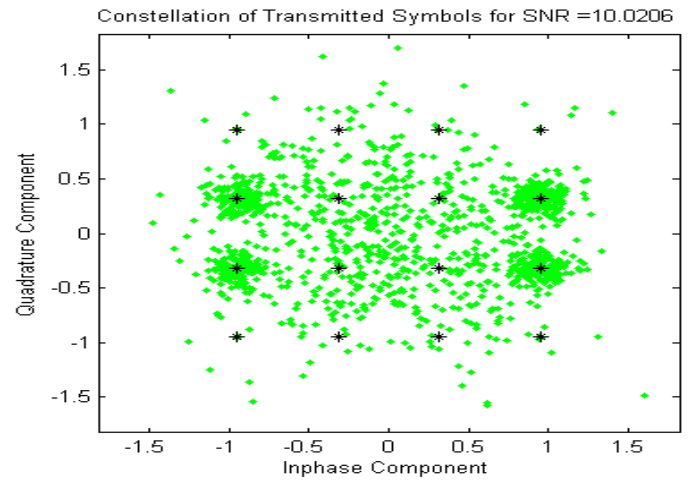

Fig.24(Constellation of transmitted symbols in case of Nakagami-m channel with $m=3$ for SNR 10 DB for 16QAM) 


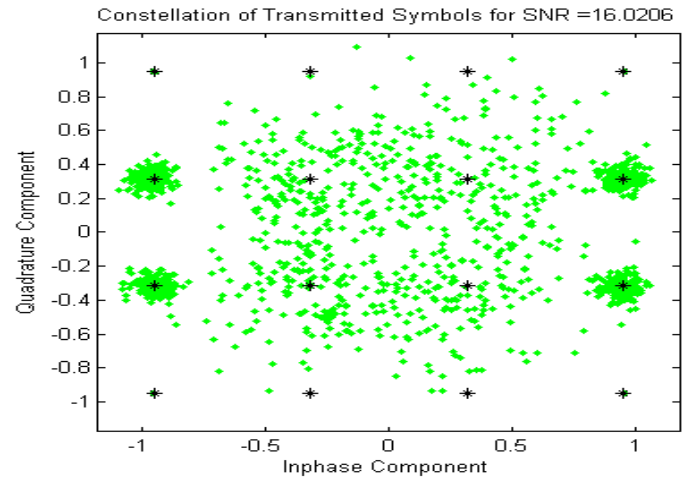

Fig.25(Constellation of transmitted symbols in case of Nakagami-m channel with $m=3$ for SNR 16 DB for 16QAM)

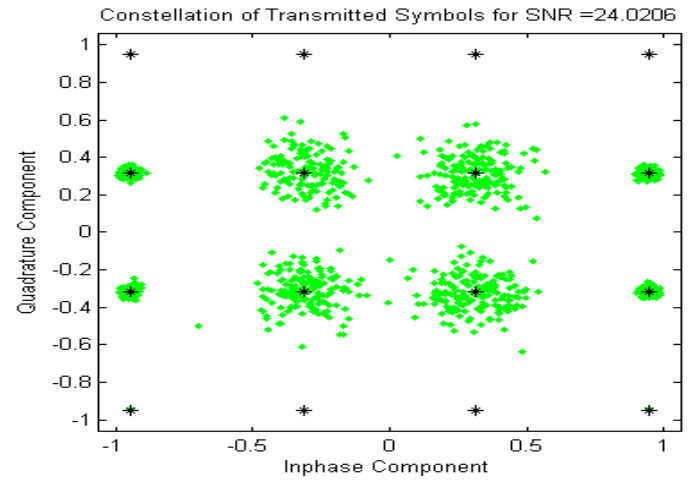

Fig.26(Constellation of transmitted symbols in case of Nakagami-m channel with $m=3$ for SNR 24 DB for 16QAM)

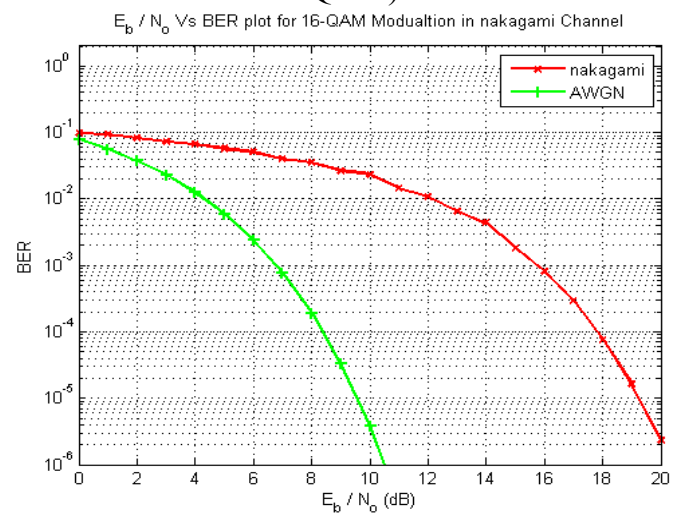

Fig.27(Comparison of BER between AWGN and Nakagami-m channel with $\mathrm{m}=3$ for 16-QAM)

Fig. 28 shows the constellation of transmitted symbols for nakagami-m fading channel with value of nakagami factor $\mathrm{m}=4$ at SNR $6 \mathrm{DB}$ and achieves BER of order $10^{-1}$.

Fig.29 shows the constellation at SNR 10 DB and achieves BER of order $10^{-1}$.Fig.30 shows the constellation at SNR 16 DB and achieves BER of order $10^{-2}$.Fig.31 shows the constellation at 20 DB SNR and BER is of order $10^{-3}$.Fig. 32 shows the constellation at $22 \mathrm{DB}$ SNR and BER is of order $10^{-4}$ but transmitted signal is still faded as seen in figure constellations are dispersed and we need more SNR to transmit the signal.

Fig. 33 shows Comparison of BER between AWGN and Nakagami-m channel with $m=4$ for $16-\mathrm{QAM}$.It shows that we need $15 \mathrm{DB}$ more SNR in case of nakagami-m fading channel with $\mathrm{m}=4$ to achieve the same BER of AWGN. 


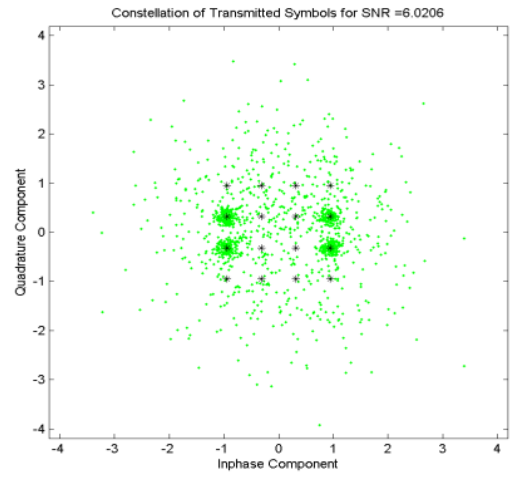

Fig.28(Constellation of transmitted symbols in case of Nakagami-m channel with $m=4$ for SNR 6 DB )

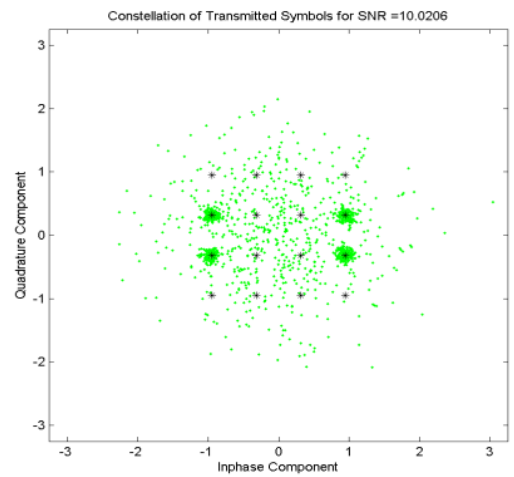

Fig.29(Constellation of transmitted symbols in case of Nakagami-m channel with $m=4$ for SNR 10 DB for 16-

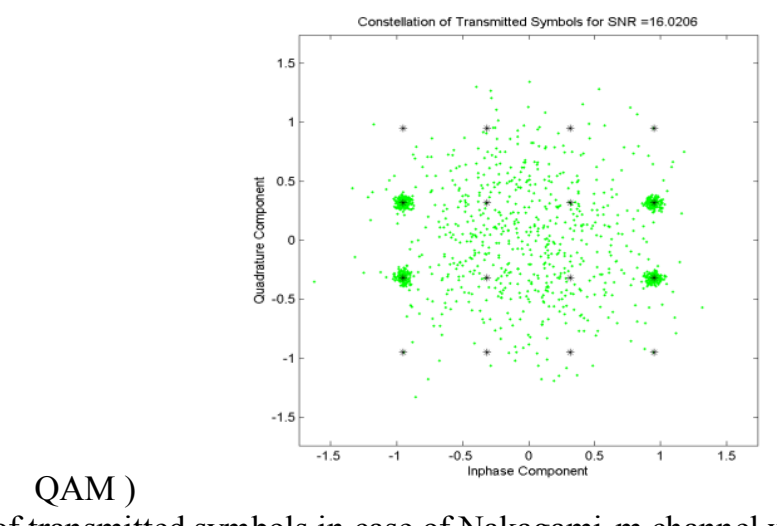

Fig.30(Constellation of transmitted symbols in case of Nakagami-m channel with $m=4$ for SNR 16 DB for 16QAM)

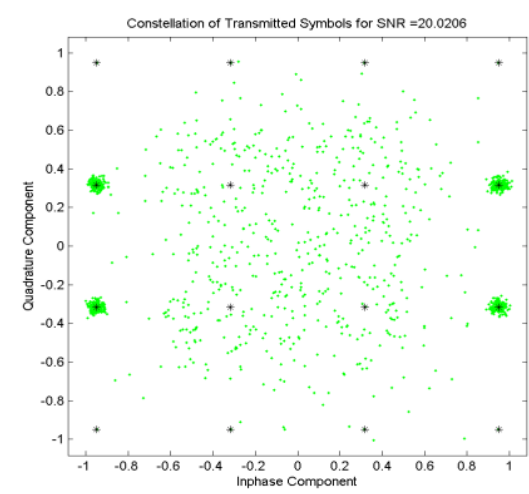

Fig.31(Constellation of transmitted symbols in case of Nakagami-m channel with $m=4$ for SNR 20 DB for 16QAM) 


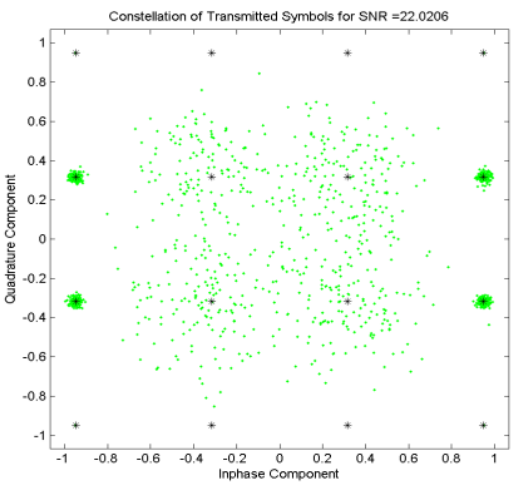

Fig.32(Constellation of transmitted symbols in case of Nakagami-m channel with $m=4$ for SNR 22 DB for 16QAM)

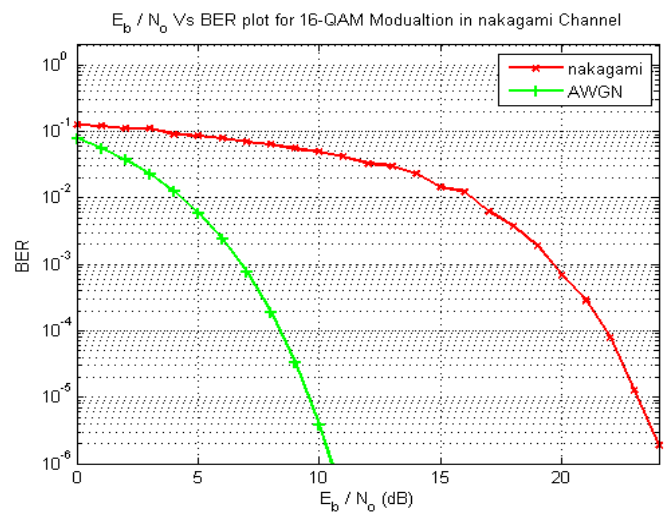

Fig.33 (Comparison of BER between AWGN and Nakagami-m channel with $\mathrm{m}=4$ for 16-QAM)

Fig. 34 shows the constellation of transmitted symbols for nakagami-m fading channel with value of nakagami factor $\mathrm{m}=5$ at SNR $6 \mathrm{DB}$ and achieves BER of order $10^{-1}$.

Fig.35 shows the constellation at SNR $10 \mathrm{DB}$ and achieves BER of order $10^{-1}$. Fig.36 shows the constellation at SNR $16 \mathrm{DB}$ and achieves BER of order $10^{-1}$. Fig.37 shows the constellation at 20 DB SNR and BER is of order $10^{-2}$. Fig. 38 shows the constellation at $26 \mathrm{DB}$ SNR and BER is of order $10^{-4}$ but transmitted signal is still faded as seen in figure constellations are dispersed and we need more SNR to transmit the signal.Fig. 39 shows the constellation at $30 \mathrm{DB}$ SNR and achieves BER of order $10^{-6}$.

Fig.40 shows Comparison of BER between AWGN and Nakagami-m channel with $\mathrm{m}=5$ for 16-QAM .It shows that we need $20 \mathrm{DB}$ more SNR in case of nakagami-m fading channel with $\mathrm{m}=5$ to achieve the same BER of AWGN.

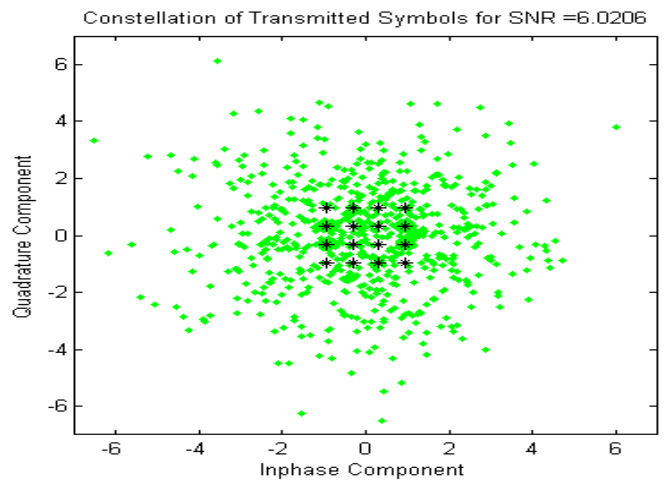

Fig.34(Constellation of transmitted symbols in case of Nakagami-m channel with $\mathrm{m}=5$ for SNR 6 DB for 16QAM ) 


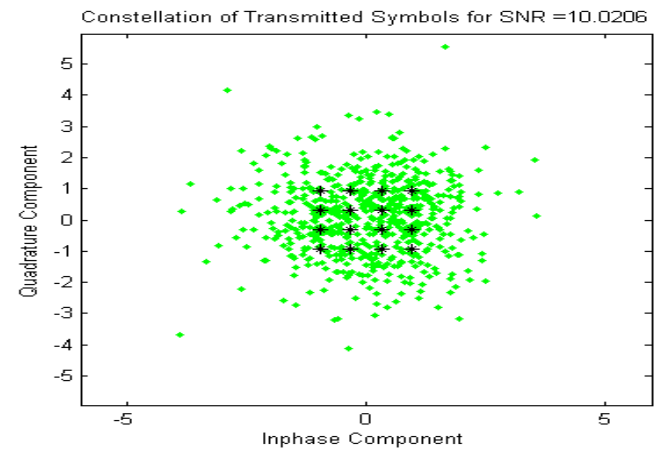

Fig.35 (Constellation of transmitted symbols in case of Nakagami-m channel with $\mathrm{m}=5$ for SNR 10 DB for 16QAM)

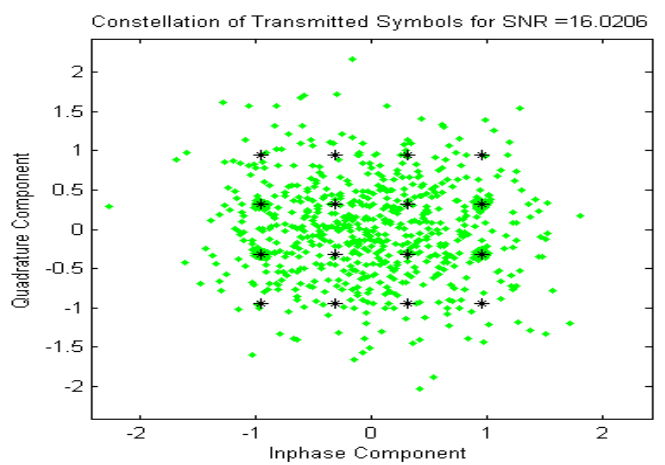

Fig.36 (Constellation of transmitted symbols in case of Nakagami-m channel with $\mathrm{m}=5$ for SNR 16 DB for 16QAM)

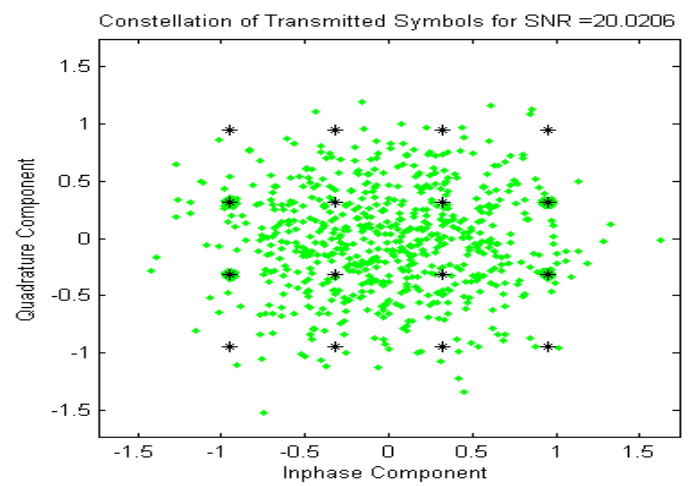

Fig.37(Constellation of transmitted symbols in case of Nakagami-m channel with $m=5$ for SNR 20 DB for 16QAM)

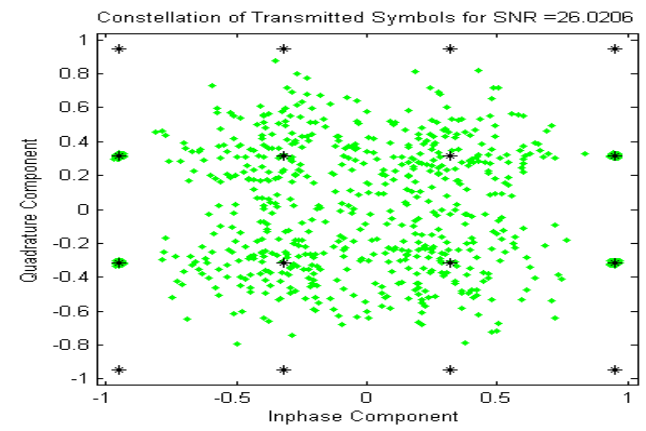

Fig.38(Constellation of transmitted symbols in case of Nakagami-m channel with $m=5$ for SNR 26 DB for 16QAM) 


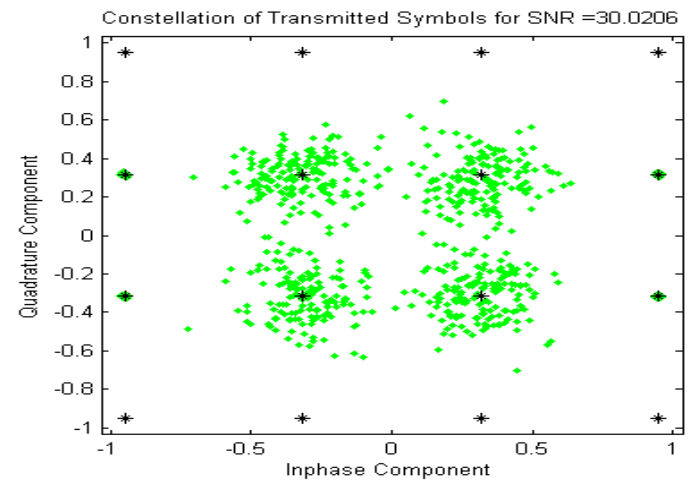

Fig.39(Constellation of transmitted symbols in case of Nakagami-m channel with $m=5$ for SNR 30 DB for 16QAM)

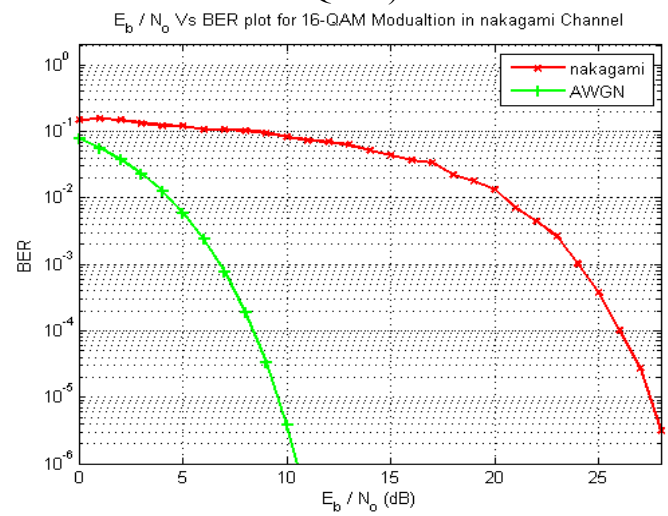

Fig.40 (Comparison of BER between AWGN and Nakagami-m channel with $m=5$ for 16-QAM)

Fig.41 shows a comparison of BER for nakagami-m fading channel with varying the value of nakagami factor $m=1$ to $m=5$ for 16-QAM technique. As seen in figure when the value of $m$ is increasing then the effect of fading is also increasing and we need more SNR to transmit the signal to overcome the effect of fading.

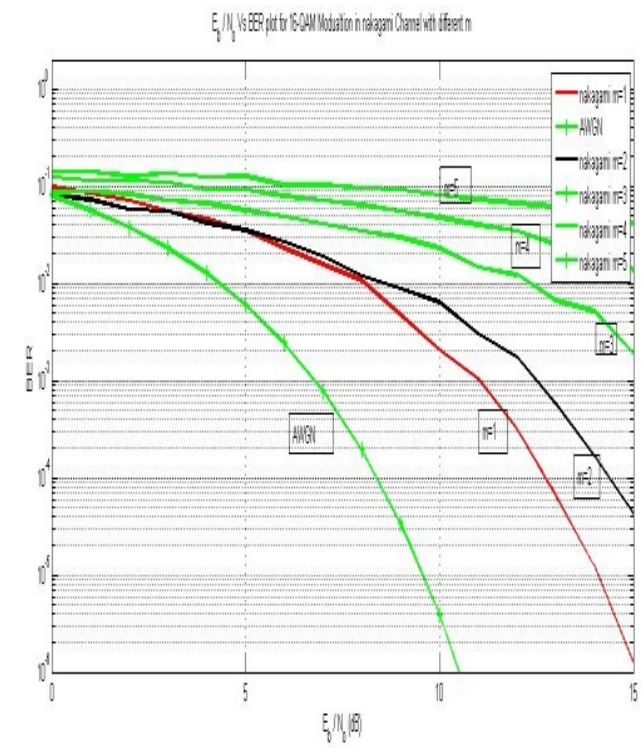

Fig.41 ( Comparison of BER for Nakagami-m channel with $m=1$ to 5 for 16-QAM)

Table 1 shows a comparison of BER for different values of SNR for nakagami parameter $m$ varies from $m=1$ to $\mathrm{m}=5$.It shows that when nakagami parameter $\mathrm{m}$ increases then it requires more SNR to reduce the BER.

\begin{tabular}{|c|c|c|c|c|c|}
\hline SNR(Db) & $\mathbf{m}=\mathbf{1}$ & $\mathbf{m}=\mathbf{2}$ & $\mathbf{m}=\mathbf{3}$ & $\mathbf{m}=\mathbf{4}$ & $\mathbf{m}=\mathbf{5}$ \\
\hline 6 & & & & & \\
\hline
\end{tabular}


Study and Comparison of Constellation diagrams of 16-QAM in different fading channels

\begin{tabular}{|c|l|l|l|l|l|}
\hline 10 & $10^{-3}$ & $10^{-2}$ & $10^{-2}$ & $10^{-1}$ & $10^{-1}$ \\
\hline 16 & $10^{-6}$ & $10^{-5}$ & $10^{-3}$ & $10^{-2}$ & $10^{-1}$ \\
\hline 20 & & & $10^{-5}$ & $10^{-3}$ & $10^{-2}$ \\
\hline 22 & & & $10^{-6}$ & $10^{-4}$ & $10^{-3}$ \\
\hline 24 & & & $10^{-6}$ & $10^{-5}$ & $10^{-3}$ \\
\hline 26 & & & & $10^{-6}$ & $10^{-4}$ \\
\hline 30 & & & & & $10^{-6}$ \\
\hline
\end{tabular}

Table I. (comparison of ber of nakagami-m fading channel)

\section{Conclusions}

In this paper, we simulated the 16-QAM modulation scheme over Rayleigh and nakagami-m fading channel and draw its constellation diagram to better understand the effect of fading with varying SNR. We also find the order of BER for Rayleigh and nakagami-m fading channel with varying $m$ parameter $(m=1$ to $m=5)$ and compare it with the BER of AWGN channel. Rayleigh fading channel need more SNR to obtain the same BER as AWGN channel. It is found that in nakagami-m channel the BER is increased with increasing $\mathrm{m}$ parameter value and it need more SNR to transmit the signal to obtain the same BER as AWGN channel.

\section{References}

[1] T. S. Rapport, Wireless Communication: Principles and Practice, $2^{\text {nd }}$ ed., Pearson Education.

[2] J.G. Proakis, Digital Communication, $4^{\text {th }}$ ed. New York: McGraw-Hill,2001

[3] Y. G. Kim and N. C. Beaulieu, "S+N Energy Selection Combining For MPSK and 16-QAM Signaling in Nakagami-m and Rician Fading Channels" IEEE Trans. Comm. Vol. 59, No. 2, Feb. 11

[4] M. K. Simon and M. S. Alouini, Digital Communication over Fading Channels: A Unified Approach to Performance Analysis, $2^{\text {nd }}$ ed. New York: Wiley, 2005.

[5] Win M. Z. and Chrisikos, G., "MRC Performance for M-ary Modulation in Arbitrarily Correlated Nakagami Fading Channels", IEEE Comm. Letters, (2000).

[6] Du, Z., Cheng, J. and Beaulieu, N. C., "Error Rate of OFDM Signals on Frequency Selective Nakagami-m Fading Channel", IEEE Comm. Society Globecom : ,3994-3998(2004).

[7] Y. S. Cho, J. Kim, W. Y. Yang and C. G. Kang,"MIMO-OFDM Wireless Communication with MATLAB”,IEEE Press and John Wiley \& Sons(Asia) Pte Ltd.

[8] Y. G. Kim and N. C. Beaulieu, " S+N Energy Selection Combining For BPSK Signaling in Rayleigh Fading Channels", IEEE Trans. Comm. Vol. 58, No. 1, Jan. 10.

[9] Z. Luo and F. Hu, "Simulation Models for Independent Rayleigh Fading Channels",IEEE Mobile Congress(GMC),2010 Global: ISBN-978-1-4244-9003-5.

[10] E. A. Neasmith and N. C. Beaulieu, "New Results on Selection Diversity” IEEE Trans. Comm. Vol. 46, No. 5, May. 1998.

[11] Y. G. Kim and S. W. Kim, " Optimum Selection Diversity for BPSK Signals in Rayleigh fading Channels" IEEE Trans. Comm. Vol. 49, No. 10, Oct. 2001 